\title{
Production of Tightly Focused E-Beams with High-Current Accelerators*
}

\author{
J. W. Poukey. M. G. Mazarakis, \\ C. A. Frost. and J. J. Ramirez \\ Sandia National Laboratories \\ Albuquerque, NM 87185
}

\begin{abstract}
Using numerical modeling we study several approaches to the problem of designing an injector to produce a 3-30 kA, 2-4 mm diametcr electron beam in the energy range $10-20 \mathrm{MeV}$. The cathode may be small in diameter and immersed in a strong magnetic field, producing an equilibrium beam for transport to a target (the "immersed" case). This approach appears to be the most promising for applications such as radiography, and we shall emphasize it in this paper. The alternative is the conventional "non-immersed" cathode. in which the beam from a larger-radius, cold-beam cathode is focused with magnetic lenses to a small spot on the target. Because the non-immersed case has been extensively studied. and because it has disadvantages for our purposes. we shall only discuss a few of our non immersedcathode injector studies, primarily for purposes of comparison.

Either type of diode is to be powered by an inductive voltage adder based on the successful SABRE/Hermes III/RADLAC (SMILE) magnetically-insulated-transmissionline design concepts. ${ }^{1,2}$ A possible variation uses a re-entrant geometry with low electric stresses so that only the cathode face emits. We discuss issues such as dumping excess current and voltage dependence of the focus.
\end{abstract}

\section{INTRODUCTION}

The problem of producing small-diameter electron beams at high voltages (10-20 MV) and currents $(3-30 \mathrm{kA})$ is of interest for several applications. including radiography. There are two hasic issues which we will consider. namely the method for accelerating the beam.

*This work was supported by U.S. D.O.E. 0-7803-0135-8/91\$03.00 OIEEE and the design of the injector. The conventional method ${ }^{3.4}$ uses a standard multi-gap linac with a non-immersed cathode in the injector diode. Code calculations for the FXR system, including the diode, the transport and acceleration through 48 gaps, and the final focus, have been described by Boyd. ${ }^{4}$

In our approach to beam acceleration. we propose an MITL (magnetically insulated transmission line) voltage adder of the Hermes III/SABRE type ${ }^{1,5}$ to apply the entire voltage (e.g., $10 \mathrm{MV}$ ) across a single electrondiode gap $\mathrm{d}$. The primary advantages of this approach, as compared to the conventional linac, are: (1) significant reduction in cost. (2) substantial reduction in complexity. and (3) avoidance of instabilities such as BBU (beam breakup).

In our approach to injector design. we propose the immersed diode. The primary advantage of this diode, as we shall show. is relative insensitivity to variations in applied voltage and $B$ field. Another advantage for some applications is the production of a highcurrent. small-radius beam in equilibrium, as opposed to a beam focused at only one axial location.

\section{IMMERSED DIODES}

Using the 2-D electromagnetic PIC code MAGIC, we have simulated a number of immersed diodes (see Table I). An example (Run 13) at the SABRE voltage of $10 \mathrm{MV}$ is shown in Fig. 1. The idea is to create a heam from a small-radius $\left(r_{k}\right)$ tip inside a large $B$, field. If $B_{2}$ is large and uniform enough from cathode to target. the electrons will follow the $B$ lines and the size of the beam at the target will be about $r_{k}$.

The motivations for this approach. as opposed to non-immersed systems with magnetic lenses, ${ }^{6}$ are: (1) The successful 
IBEX experiments ${ }^{7}$ (see run 0 in Table I). By "successful" is meant production of a small. low-emittance beam, and agreement between MAGIC and measurement. (2) The relative insensitivity of beam parameters at the target, namely $r_{\mathrm{h}}$ (beam radius) and $\beta_{\perp}$ (transverse velocity). to variations in voltage $V$ and applied $B_{7}$ (see Table I). We also varied drift tube radius $r_{1}$ (compare runs 13 and 15), but found almost no change in results. There is some variation with $B_{7}$, as seen in runs 9,13 and 12.17 ; as expected, the higher $B_{z}$ results in a higher quality beam.

For comparison, we did a series of quasistatic runs using a trajectory code for a non-immersed diode with a magnetic lens. We used a large-cathode, $d=70 \mathrm{~cm}$ (A-K gap) system which emits from a flat velvet region of radius $6 \mathrm{~cm}$. We varied $V$ and $B_{z}$ by $\pm 10 \%$ about values for a good focus (best result: beam diameter of $2 \mathrm{~mm}$ ). and found that the focus moves in axial position $z$ by enough to cause increases in $r_{b}$ of up to $4 \mathrm{~mm}$. This does not preclude using such a diode, but one must control $\mathrm{V}$ and $\mathrm{B}$, to much greater precision than for our immersed cases.

Table 1

Immersed Diodes for Radiography. In all cases. except $0, A-K$ gap $d=15 \mathrm{~cm}$, and the applied $B_{7}$ is uniform. In case 0 , the IBEX experiment, ${ }^{7}$ the $A-K$ gap was $d=7 \mathrm{~cm}$. The last three columns are the output beam at the target. Code: MAGIC. Run 13 is shown in Fig. 1.

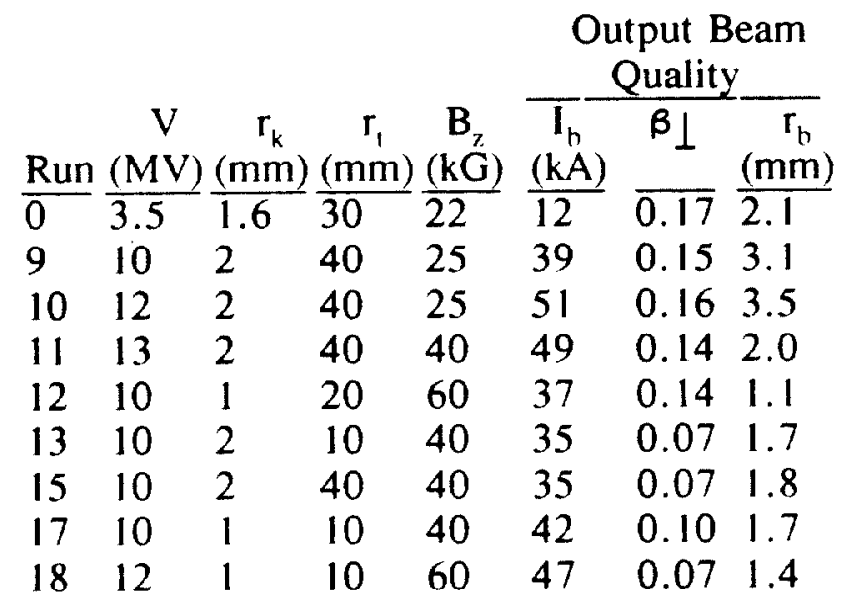

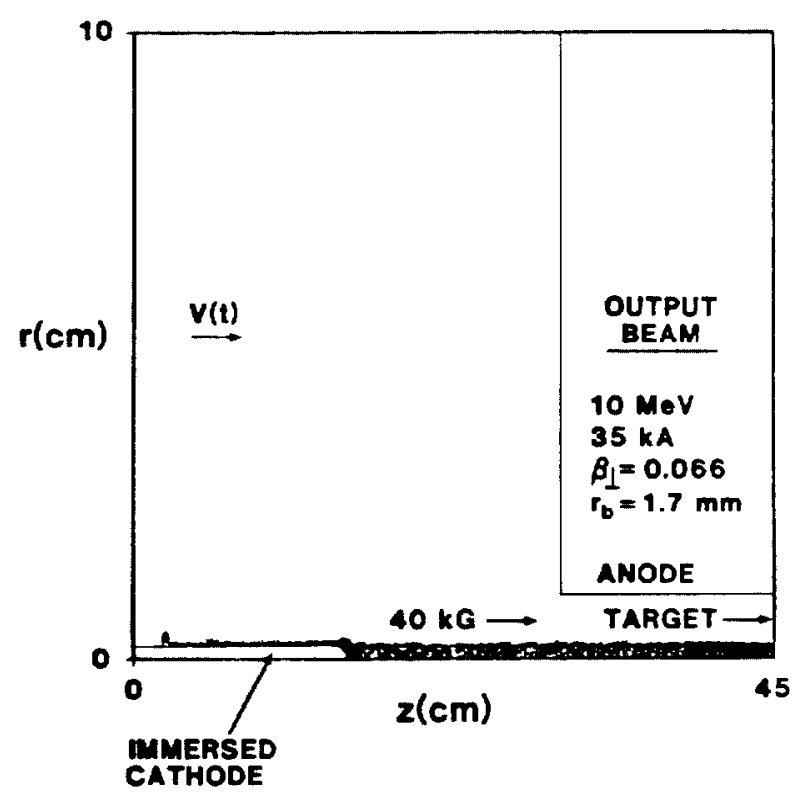

Figure 1. MAGIC simulation of immersed diode at $10 \mathrm{MV}, 35 \mathrm{kA}$, (see run 13 of Table I). The entire diode and target (rhs) are immersed in $40 \mathrm{kG}$, yielding a high quality. small-diameter beam.

\section{TRANSITION TO VOLTAGE ADDER}

The input "voltage pulse" in Fig. 1 is generated by an MITL voltage-adder system such as SABRE. 5 Some simulations of the entire MITL and ten feeds have been attempted, but here we just discuss the transition from the adder to the diode. A sample run is given in Fig. 2, which includes the large outer conductor from the coaxial feed, and a truncated small-radius cathode. The coils produce $60 \mathrm{kG}$ for the immersed diode, and allow over half the total of $84 \mathrm{kA}$ to be "dumped" radially.

The main problem here is that the configuration inevitably yields some "halo" electrons. originating back on the cathode shank. In Fig. 2, these lead to an rms beam radius $r_{b}$ on target of $2.8 \mathrm{~mm}$; this is somewhat larger than desirable. Using a more gentle taper reduces this radius somewhat. but the problems of MITL sheath and shank electrons persist. Possible solutions include current-dump projections in the MITL. aperturing, and contouring the cathode to follow a flux surface. 


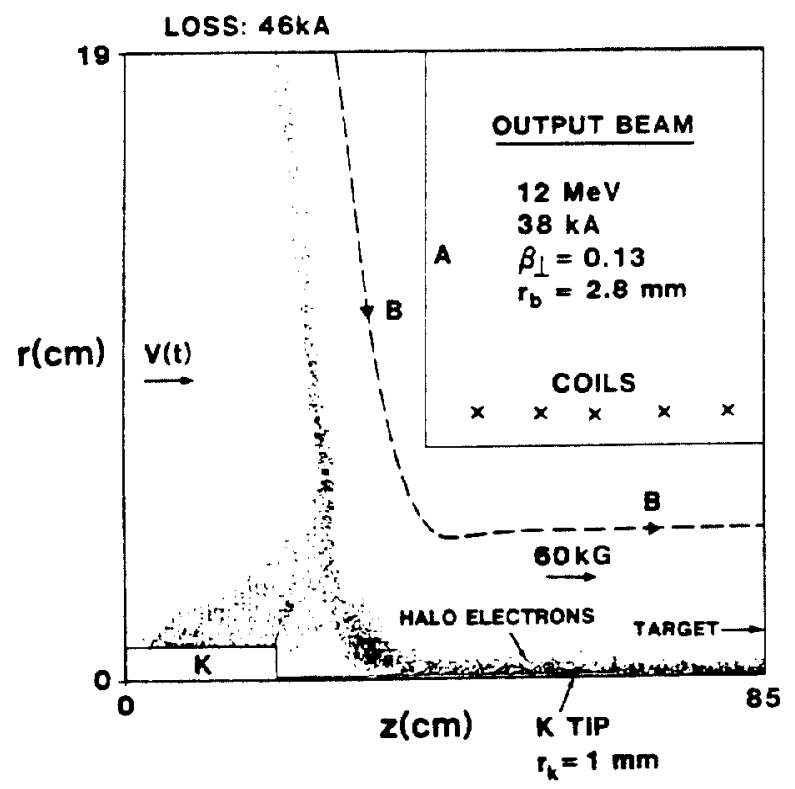

Figure 2. MAGIC run of immersed diode plus transition to voltage adder. The adder gives input voltage $V(t)$ (modeled as TEM wave; $\max 12 \mathrm{MV}$ ). Note loss along fringing B lines.

\section{OTHER SYSTEMS}

We have also studied non-immersed diodes with magnetic lenses in some detail. As discussed above, we find that such diodes are not as good as immersed ones for our purposes (e.g., radiography), due to the difficulty in maintaining a very small focal spot size over realistic voltage pulses.

An interesting variation here is a nonMITL voltage adder of the Recirculating Linear Accelerator type, ${ }^{8}$ with a carefully designed, low-stress, non-immersed cathode and a re-entrant anode containing magneticfield coils. Both MAGIC and trajectory-code runs show some good properties of this system, although voltage sensitivity may still be a problem. Further studies would be needed to investigate this point, and certainly to achieve a practical design.

\section{SUMMARY}

For the production of small-diameter electron beams in the range $10-20 \mathrm{MeV}$, we propose an inductive-voltage-adder, single (diode)-gap approach, e.g., SABRE instead of the conventional multi-gap linac. For the injector, we propose to use a small-diameter cathode immersed in a field of 40-60 kG. Our calculations predict that very high current densities can be expected, with relative insensitivity to parameter variations.

\section{REFERENCES}

[1] T. W. L. Sanford et al., "Dynamics of electron flow in extended planar-anode diode operating at $19 \mathrm{MV}$ and $700 \mathrm{kA}$." J. Appl. Phys., vol. 67, pp. 1700-1711, Feb. 1990.

[2] M. G. Mazarakis et al., "SMILE, a new version for the Radlac Il Linac," in Proc. 1990 Linac Conf., Albuquerque, NM, Sept. 1990. pp. 438-440.

[3] T. P. Hughes et al., "Diode and transport calculations for REX, DARHT, and PHERMEX," Mission Research Report MRC/ABQ-R-1244, Feb. 1990.

[4] J. K. Boyd. "Accelerator code simulation of FXR transport," in Proc. Conference on Computer Codes and the Linac Community, Los Alamos, NM, Jan. 1990. pp. $221-232$.

[5] SABRE is an upgrade of the HELIA accelerator. (D. L. Johnson and J. P. Corley, Sandia National Labs, private communication.) For HELIA and HERMES III see, e.g.. Proc. 7th IEEE Pulsed Power Conf., Monterey. CA, June 1989.

[6] R. B. Miller et al., "Matching beams from relativistic electron guns into solenoidal transport systems," J. Appl. Phys., vol. 62, pp. 3535-3538, Nov. 1987.

[7] C. A. Frost et al., "High-brightness immersed source injector characterization." in Proc. 1989 IEEE Particle Accelerator Conf.. Chicago. IL. March 1989. pp. 1456-1458.

[8] M. G. Mazarakis et al.. "Electron diodes and cavity design for the new $4 \mathrm{MeV}$ injector of the RLA." in Proc. 1990 Linac Conf.. Albuquerque. NM. Sept. 1990. pp. 605-607. 